\title{
INTEGRAL Observations of the Pulsar PSR J1846-0258
}

\author{
S. J. Sturner ${ }^{*, \dagger}$ and C. R. Shrader*,† \\ ${ }^{*}$ CRESST and Astroparticle Physics Laboratory NASA/GSFC, Greenbelt, MD 20771, USA \\ ${ }^{\dagger}$ Universities Space Research Association, 10211 Wincopin Circle, Suite 500 Columbia, MD 21044, USA
}

\begin{abstract}
We present the results of a study of the high-energy properties of PSR J1846-0258 using INTEGRAL data. PSR J1846-0258 has a period of $325 \mathrm{~ms}$ and is embedded within a PWN that is associated with the SNR KES 75. This pulsar is unusual for two reasons. First, like Geminga, no radio pulsations have yet been detected from this pulsar. It has thus far been detected exclusively in the X-ray and gamma-ray bands. Second, it has a large inferred magnetic field, $\sim 5 \times 10^{13} G$. The 20-200 keV spectra derived from $2.35 \mathrm{Msec}$ of INTEGRAL IBIS/ISGRI observations are consistent with an extension of the previous spectral results at energies below $10 \mathrm{keV}$ from Chandra. The possibility of detecting PSR J1846-0258 with GLAST is discussed as well as the implications a GLAST detection would have on pulsar theory, specifically photon splitting in the magnetospheres of high-field pulsars.
\end{abstract}

Keywords: Pulsars - PSR J1846-0258 - neutron stars - gamma rays

PACS: $97.60 . \mathrm{Gb}$

\section{INTRODUCTION}

PSR J1846-0258 is a rotation-powered pulsar located near the center of the composite supernova remnant (SNR) Kes 75 (G29.7-0.3) within a pulsar wind nebula (PWN) at a distance of $\sim 19 \mathrm{kpc}$ [1]. The pulsar's period and period derrivative are $325 \mathrm{~ms}$ and $7.1 \times 10^{-12} \mathrm{~s} \mathrm{~s}^{-1}$, respectively. This leads to a pulsar spin-down age of $\sim 723$ years [2]. Assuming magnetic dipole energy losses, the polar magnetic field strength of PSR J1846-0258 is $4.9 \times 10^{13} \mathrm{G}$, a factor of $>3$ larger than that of PSR B1509-58 and more than an order of magnitude larger than the Crab pulsar [2,3]. Like Geminga, it is radio quiet [4]. The pulsar was discovered in X-rays using data from RXTE by Gotthelf et al. [5]. Assuming a distance of $19 \mathrm{kpc}$, the nonthermal luminosity of the pulsar and the PWN are $4.1 \times 10^{35} \mathrm{ergs} \mathrm{s}^{-1}$ and $1.7 \times 10^{36} \mathrm{ergs} \mathrm{s}^{-1}$ in the $0.5-10 \mathrm{keV}$ band, respectively. These are second only to the Crab pulsar and nebula. The efficiency with which the pulsar is converting rotational energy loss to X-rays is $\sim 6 \times$ that of the Crab pulsar but is similar to that for PSR B0540-69 in the LMC [3].

Kes 75 is only $3.5^{\prime}$ in diameter. Thus X-ray observatories such as ASCA and BeppoSAX could only comment on the spatially integrated spectrum from all components, e.g. the shell, PWN, and pulsar. The integrated $<10 \mathrm{keV}$ spectrum from the entire SNR including the pulsar and PWN has been successfully fit with two component models consisting of a thermal component such as MEKAL or Raymond-Smith with $\mathrm{kT}=0.4-0.8 \mathrm{keV}$ and a nonthermal power law component with an index $\Gamma \sim 2[2,6]$.

The fine angular resolution of Chandra allowed for spectral analysis of the various sub-regions of the SNR. This led to the identification of 4 spectral components [3]: (1) a thermal component from the SNR shell $(\mathrm{kT}=0.69 \mathrm{keV})$, (2) nonthermal emission from shock accelerated electrons in the shell $(\Gamma \sim 1.72)$, (3) nonthermal emission from the PWN $(\Gamma \sim 1.92)$, and $(4)$ nonthermal emission from the pulsar PSR J1846-0258 $(\Gamma \sim 1.39)$. The relative normalizations of the PWN and pulsar components suggest that the pulsar emission should begin to dominate the spectrum at $\sim 45 \mathrm{keV}$.

\section{RESULTS \& DISCUSSION}

We have analyzed all the publicly available INTEGRAL IBIS/ISGRI data for this work. We chose only those Science Windows (SCWs) with pointing directions within $10^{\circ}$ of PSR J1846-0258. The data consisted of 1127 SCWs ranging from March 10, 2003 to October 8, 2005 with a total good time of $2.35 \mathrm{Msec}$. Data reduction was performed using the standard OSA 5.1 analysis software package available from the INTEGRAL Science Data Centre.

We fit the combined BeppoSAX MECS - INTEGRAL IBIS/ISGRI data set in XSPEC with a MEKAL+Power Law model. Given the angular resolution of these instruments, the spectra were spatially integrated over the entire SNR. We explored whether there was evidence for a change in the best-fit power law index between the MECS and ISGRI 


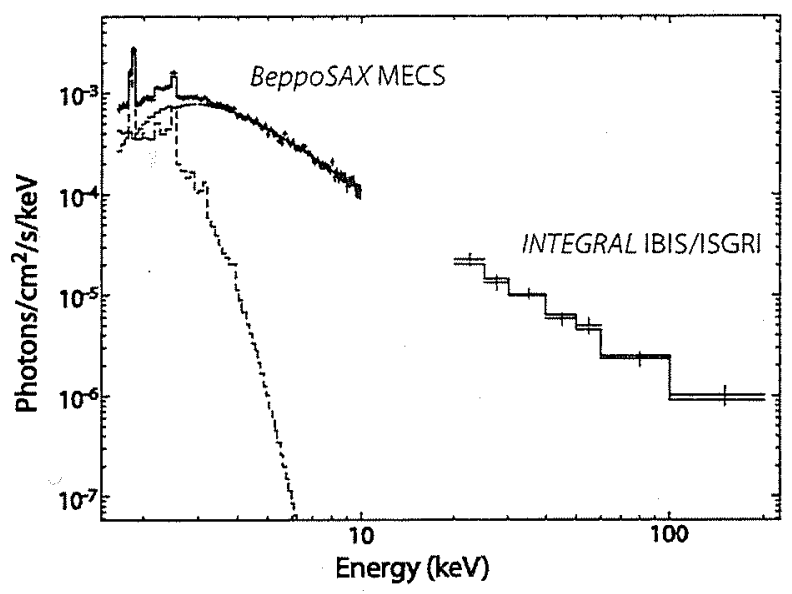

FIGURE 1. Best fit to combined BeppoSAX MECS - INTEGRAL IBIS/ISGRI data set using a MEKAL + Power Law model. See text for best fit parameters.

energy bands. We also explored whether the ISGRI data was best fit by a single power law or by the sum of two different power laws with indices of 1.39 and 1.92 as suggested by the Chandra PWN and pulsar spectra [3].

There is strong evidence that the power law index in the IBIS/ISGRI energy band (20-200 keV) is significantly harder than in the MECS energy band $(1.65-10 \mathrm{keV})$. We found that when we allowed the power law indices to vary between the two data sets, that in the low energy band the best fit power law had an index of $\Gamma=2.18_{-0.11}^{+0.09}$ versus $\Gamma=1.67_{-0.19}^{+0.23}$ in the IBIS/ISGRI band. An FTEST indicates that the reduction in $\chi^{2}$ that resulted when the power law indices were allowed to vary between the two data sets had a chance probability of occurring of only $0.33 \%$.

We also found that the ISGRI data alone was consistent with both a single power law $\left(\Gamma=1.68_{-0.20}^{+0.22}\right)$ and with a dual power law where the indices were fixed at the values found by Chandra for the pulsar and PWN $(\Gamma=1.39 \& 1.92)$. Additional data or improvements to the ISGRI analysis software will enable us to extend the spectrum to energies $>200 \mathrm{keV}$ which could allow us to better determine whether the high-energy gamma-ray spectrum is pulsar or PWN dominated.

We then explored whether PSR J1846-0258 will be detectable by GLAST. We extrapolated the best-fit $\Gamma=1.68$ and $\Gamma=1.39$ power laws using normalizations based on the IBIS/ISGRI data. We chose to test extrapolations of both of these power laws because while the Chandra results indicate that the $\Gamma=1.39$ power law from the pulsar should dominate at high energies, the ISGRI data could not differentiate between a single or dual power law model in its energy band. We then applied exponential cut offs at $100 \mathrm{MeV} .1 \mathrm{GeV}$, and $5 \mathrm{GeV}$ in order to better approximate the true pulsar spectra.

The extrapolated integrated flux $>100 \mathrm{MeV}$ for PSR $1846-0258$ ranged from $2.5 \times 10^{-7} \mathrm{~cm}^{-2} \mathrm{~s}^{-1}$ for $\Gamma=1.68$ and $E_{\text {cut }}=100 \mathrm{MeV}$ to $1.1 \times 10^{-5} \mathrm{~cm}^{-2} \mathrm{~s}^{-1}$ for $\Gamma=1.39$ and $E_{\text {cut }}=5 \mathrm{GeV}$. Taking the GLAST point source sensitivity in the Galactic Plane to be $\sim 6 \times 10^{-9} \mathrm{~cm}^{-2} \mathrm{~s}^{-1}$, we find that the pulsar emission ranges from $\sim 40-1800 \times$ the GLAST sensitivity and thus PSR J1846-0258 should be detectable by GLAST. It could be argued that a spectral cut off at energies near $10 \mathrm{MeV}$ may indeed be possible or even likely given PSR J1846-0258's high magnetic field strength $\left(B \sim 5 \times 10^{13} \mathrm{G}\right)$ and thus its similarity to another high-field gamma-ray pulsar, PSR B1509-58 $\left(B \sim 1.6 \times 10^{13} \mathrm{G}\right)$ whose spectrum is cut off below $30 \mathrm{MeV}$, possibly by photon splitting [7]. Detection of PSR J1846-0258 by GLAST would provide constraints on the photon splitting hypothesis for the spectral cutoff of high-field gamma-ray pulsars.

\section{REFERENCES}

1. Becker, R.H., \& Helfand, D.J. 1984, ApJ, 283, 154

2. Mereghetti, S., Bandiera, R., Bocchino, F., \& Israel, G.L. 2002, ApI, 574, 873

3. Helfand, D.J., Collins, B.F., \& Gotthelf, E. V. 2003, ApJ, 582, 783

4. Kaspi, V.M., Manchester, R.N., Johnston, S., Lyne, A.G., \& D'Amico, N. 1996, A.J. 111, 2028

5. Gotthelf, E.V., Vasisht, G, Boylan-Kolchin, M., \& Torii, K. 2000, ApJ, 542, L37

6. Blanton, E.L., \& Helfand, D.J. 1996, ApJ, 470, 961

7. Harding, A.K., Baring, M.G., \& Gonthier, P.L. 1997, A.pJ, 476, 246 